\title{
Quantum Measurement, Consciousness, and the Soul: a New, Alternative Position
}

\author{
Paul J. Werbos ${ }^{1}$ \\ Received: 1 February 2019 / Accepted: 25 March 2019 / Published online: 16 April 2019 \\ (C) The Author(s) 2019
}

\begin{abstract}
This paper presents a new position on the fundamental questions about quantum measurement, consciousness, and soul, which contradicts at least one sacred assumption of every major player in today's debates. It starts from a first person viewpoint, which may be characterized as Rational German existentialism, in the spirit of Goethe, Von Neumann, Heisenberg, and Raiffa. On that foundation, it rejects the obsolete concepts of primal ontology, supports the quest for a unified objective mathematical model of what underlies all of our experience, and concludes that we should simply not take a rigid position at this time on three possible types of "theory of everything" (1) the "multiverse" theory, according to which, we live in a "multiverse," an infinite dimensional space like the proposals of Everett, Wheeler, and David Deutsch, or a similar infinite-dimensional realistic theory; (2) hard core Einsteinian theories, assuming a finite dimensional cosmos, which may well be salvageable mathematically in light of new work on quantum measurement, but which would require understanding ourselves as an odd sort of "shadows of Plato's Cave"; (3) something much weirder, like random graphs, "digital universe" or cosmos as a vast neural network (of what telos?). It rejects the idea that quantum measurement has any connection to the emergent phenomena of consciousness or the soul. Further work building on this foundation rejects the classic formulation of noosphere from De Chardin and Vernadsky, but proposes that an entire species of noospheres permeating our cosmos is plausible under (1) and (2), and may in fact be the most plausible basis for explaining veridical weird experience for those of us who find it inescapable.
\end{abstract}

Keywords Quantum measurement · Consciousness · Multiverse · Realism · GHZ states · Existentialism · Time-symmetric physics · Qualia

\section{Introduction}

It is well known in neuroscience that intense hormones usually suppress the higher centers of the brain, causing something of a regression in thought and in behavior. At present, this phenomenon is especially visible in politics, where people are driven to extreme, emotional polarization, in a way which couples policy issues together in ways which make no sense objectively. Why, for example, should support for the Old Testament and even for stoning outsiders be coupled to the worldview expressed in Ayn Rand's classic novel Atlas Shrugged?

Global debates on consciousness, quantum theory, and the soul have also aroused intense emotions, and created couplings in our culture which are not there in reality. This paper will try to present a

Paul J. Werbos

paul.werbos@gmail.com

1 Department of Mathematics, University of Memphis, 5304 1st P1 N, Arlington, VA 22203, USA worldview on these issues which will probably be offensive to all of the major spokesmen for today's positions, in part, because it combines some elements from all of them, but in part, because it starts from a first person foundation which is very idiosyncratic in some respects (although there are many paths up the same mountain).

For the sake of coherence and intelligibility, the paper will begin by stating what the first person foundation is, and moving step by step through the points raised in the abstract.

\section{Brief Summary of Rational German Existentialism}

\section{The Starting Point, from Church to Bertrand Russell, Nietzsche, Confucius, and Freud}

First, let me state the underlying assumptions from more than 50 years ago which led me to this position in later years, after decades of cross-disciplinary research. 
The deepest assumptions or axioms which any scientist or philosopher builds upon cannot be proven or disproven by the use of words alone or of value-free general logic (Kant 1999; Werbos 2012).

Logic alone cannot answer the most central questions of philosophy, about the meaning of life or even just the proper way to learn things. When I tried to do this at age 15, while taking the graduate course of Alonzo Church on logic, I received a letter from Bertrand Russell summarizing the problem quite simply, roughly: "No system of axioms which do not already include words like 'good' or 'should' as axioms can possibly deduce a valid conclusion using such words." But then I realized I cannot use logic or any valid kind of reason to answer the question "What should I do?". But I can use my mind, and all of its faculties, to ask "What would I do if I were wise? More precisely, what answer would fully satisfy me after I consider all aspects, using all my faculties?"

This led to a starting point in life which I now sometimes call "the first person scientific method" (Werbos 2015). It simply entails a full self-awareness or consciousness of what I am doing as a human individual: learning directly about the vast stream of feelings coming to me (sensory feelings, echoes of my own actions, inner feelings, and a direct sense of "this good" and "this bad" (Nietzsche 1956) or "this light" and "this dark"), and sincerely trying to make sense of them, directly, using the full gamut of tools available to me, from direct sensitivity to mathematics, in an integrated way, and also trying to develop concepts and strategies for how to act on them. This viewpoint is essentially just a development of the concept of "integrity" (zhengqi) developed by Confucius and Meng Tzu, and of the concept of "sanity" implicit in the work of Freud (Werbos 2015).

As I began to apply this approach to guiding my own life, three further refinements came into the picture.

\section{First Refinement: Assimilation of Von Neumann, Raiffa, and Hebb}

First, in the realm of ethics (the larger framework which epistemology contributes to, just as the predictive thalamocortico-thalamic loops in the brain are a subsystem of the larger intelligent decision and control performed by the whole brain (Werbos 2012; Werbos and Davis 2016)), I wanted a more concrete metric or articulation of my sense of "this good" and "this bad", more powerful in guiding action than the somewhat loose and even dangerously erratic and incoherent thoughts and behavior which one could see in some of the followers of Nietzsche or Ayn Rand (2005). Thus I came to see the concept of cardinal utility function (Von Neumann and Morgenstern 2007) and the related concept of first person, subjective probability estimates (Raiffa 1968), as key foundations required for full self-awareness and sanity, even at the most basic mundane level. I also came to understand the general concept of neural network mathematics in particular (Hebb 1949) as a tool both for more accurate self-awareness and for attaining the full potential effectiveness of myself and of others in using the full faculties of mind (Werbos 2012). More recently, I wonder how close my developments before college mirrored a turning point in the life of John Stuart Mill (Micale and Micale 2009), when he continued to be a utilitarian but restructured what this meant to him in personal terms.

\section{Second Refinement: from Hebb to Serious Questions and Study of Psi}

Second, as an undergraduate at Harvard, I opened myself up to feeling the full power of many types of intelligent "classical" or "modern" Western music, and I even tried at times to observe "from afar" the workings of my own brain, as one way to get clues about the questions of functional neuroscience which I pursued ever more intensely. Probably that is why I had a kind of shock in my first person experience in March 1967 (Werbos 2006, 2018), a shock which forced me to reassess my probability assessment regarding paranormal or psychic or spiritual experience. Previously, I had agreed exactly with Hebb's assessment (Hebb 1949) that the probability that such things are real is very tiny; however, in the face of veridical facts utterly inescapable to me personally (supported by previous experiences I remembered but had discounted or repressed), I decided it was then 50-50.

Having open eyes, and even experimenting some, I then had many further experiences culminating in the common kind of experience which Greeley and McReady talk about (Greeley and McCready 1975; Krishna 2017), which pushed my personal subjective probability to more than $99 \%$ that this stuff is real, that it is central to my efforts to understand life, and that I needed to read very widely to learn whatever I could from others (still preserving proper scientific skepticism and working to understand human biases). I now view my state of mind in 1964 as only a kind of first-stage zhengqi; the full integration of that with first personal psi inputs is second-order integrity (Nietzsche 1956), following the concepts of Alchemical Marriage, the very core of the Rosicrucian Manifesto of the 1600s.

I now view the first-stage version of zhengqi, taught by narrow and feudalistic teachers like $\mathrm{ZhuXi}$, as a weak and incomplete reflection of the original ideas of Confucius and Meng Tzu, which included a full and direct study and use of many levels of "qi" (Fung 1967). In a loose and practical sense (Hegel 1861), Mao Tse-Tung may be understood as the essential spiritual antithesis to $\mathrm{ZhuXi}$, opening the door to a more complete understanding of Meng Tzu and the greater form of zhengqi, and a hope for a more sustainable culture and society.

Second stage zhengqi naturally drove me to apply first person science more energetically to making sense of the realm of psi and of the mind, but also drove me to working 
in a scientific way on the question: "How could anything like this be physically possible?" I did not want to be like those people interviewed by Greeley (Greeley Greeley and McCready 1975; Fromm 1976) who simply regressed to primitive thinking, and gave up on the search for more coherent, complete, and general (cosmopolitan) understanding.

\section{Third Refinement, Quantum Physics: From Monism to Dualism/Stapp to Dual Aspect Monism}

In the summer of 1968, working at the Rand Corporation (an experience which reaffirmed my conclusion that reality is truly "weird" by normal standards), I read my first text on quantum physics, followed by intense further courses as part of the Harvard $\mathrm{PhD}$ program in applied mathematics.

Before that, like Hebb and like Einstein (and like 'tHooft today (Hooft 2014)), I had a reasonably strong belief in the concept of objective reality. More precisely, I accepted "Einsteinian realism," which I hereby define as the belief that absolutely everything which exists in our cosmos, everything which we can ever know about, is simply the emergent outcome of the operation of certain unknown nonlinear partial differential equations (PDE) over a finite dimensional spacetime. This was not part of my philosophical axioms, nor was it part of Einstein's. (Some historians have written that (Kant 1999) was Einstein's favorite book at one point in his life.) Rather, after hundreds of years of progress in Western mathematical physics, this seemed like the natural obvious end point to expect. Even my experience in March 1968 had not changed that. This idea that we live in just one reality, one universe, is one form of the ancient philosophy of "monism," debated for millennia in India (Hume 1921).

But then I read about the starting point and work of Heisenberg and Niels Bohr (whom my wife says is a distant cousin, from the same esoterically inclined region near Trier). They too were obviously rational German existentialists, at least to the level of stage one. Heisenberg reminded us rational German existentialists that theories of physics are really just part of our efforts to predict the stream of experience ever more completely and ever more precisely. (We hold that it is not natural to just retreat into fuzzy gibberish when a better alternative is visible to us.) In this view, a theory of physics could be a theory about how objective reality works, but it does not necessarily have to be such. Einsteinian realism is just one of the options available to us. Heisenberg's version of quantum mechanics was a different option.

Henry Stapp explained the viewpoint of Heisenberg to neuroscientists as follows (Stapp 1998): "The core idea was that the basic physical theory was no longer directly about a physical world that was conceived to exist apart from anyone's knowledge of it." It offered what he called a pragmatic approach, predicting how consciousness as a kind of higher reality would have impact on the physical universe. In his most highly cited paper (Stapp 2004), he elaborated on the concept of qualia, the concept he is most famous for in debates on consciousness. "The central question is then: Why is the actualizing of this integrated pattern of activity felt as the perceiving of the triangle? More generally: Why do brain events fed the way they do? Qualia; The Experiential or Felt Quality of Actual Events." This stimulated a great debate focusing the nature of qualia, the first person subjective experiences of the mind, fully in harmony with the efforts of Goethe and of Nietzsche, but in a more scientific framework. This new viewpoint is a form of dualism, also debated for millennia (Hume 1921).

Nevertheless, Einsteinian realism and a new form of realism (which I call "Fock space realism") are also still options which we are allowed to consider when we take the pragmatic approach. If we build our physics on the foundations defined by Von Neumann (Von Neumann and Morgenstern 2007) and Raiffa (Raiffa 1968)), we will always remember that we ourselves do not really know which option is ultimately true, and that it is rational to pay serious attention to very different options or possibilities at one time. Taking the pragmatic approach, we may still follow the German existentialist approach by focusing intense personal effort to understand qualia, through "first person science," pursued in parallel with the third person effort to understand the ultimate laws of physics (Werbos 2015). These versions of realism are formally an example of "dual aspect monism," in which we still assume the existence of one mathematical reality, but give fundamental respect to the first person and third person viewpoints, which are equally fundamental to us as people.

The integration of these viewpoints is also important; however, because the emphasis of this special issue is on the ultimate laws of physics, the rest of this paper will focus almost entirely on the third person aspect.

In the pragmatic viewpoint, either in Heisenberg's option or Einstein's or in new possibilities, we define a theory of physics as a body of mathematical assumptions which we use to make predictions of experiments we can perform and replicate in physics. Many philosophers have attacked that approach by saying that all such theories are inherently incomplete. They do not "explain" why the best available predictive theory of physics "happens to be true ontologically." People who take that too seriously are confused about the very nature of science. Yes, it is rational to look for a deeper mathematical theory to explain why the current theory emerges as a good approximation (just as Einstein did to Newton with gravity), when we might find empirical evidence to justify it, but it is irrational to be unhappy with the need for a theory in which the ultimate level of "substance" or "reality" is just a set of mathematical constructs like fields or functions defined over some mathematical space. Those philosophers who publish rants about "physicalism" in modern science and then suggest that consciousness must be explained by some physical 
substance like psychic phlogiston or moola praloola are grossly unaware of what physics is today. They are like the people who assumed that electromagnetic waves must be propagating over some kind of ether; that kind of physicalism was replaced by mathematicalism long ago, when people came to really appreciate Maxwell's Laws.

Modern culture has fractured very deeply, and failed to achieve its own integrity, in the study of quantum mechanics. One stream, rooted more in academic philosophy than in physics, strives to develop ontological interpretations of quantum mechanics $(\mathrm{QM})$, which is frequently assumed to be just one established mathematical theory yielding predictions which everyone agrees on, developed in the 1920s. (For example, even in this century I have heard Aharonov emphasize in talks to NSF and elsewhere that his version of time symmetric physics as of that time would yield predictions identical to those of traditional QM.) Another stream, rooted more in the effort to predict and model empirical data and to design new systems (Dowling et al. 2007), gives first priority to understanding and using just two leading mathematical theories (Weinberg 1995) about how to predict experiments in physics: (1) canonical quantum field theory (QFT), still the primary mainstay of electronics and photonics, the great breakthrough of the 1950s which resulted in a Nobel Prize for Schwinger, Feynman, and Tomonoga; (2) Feynman path QFT, the mainstay today of high energy and particle physics. A third stream, rooted in rigorous mathematics (Glimm and Jaffe 1981; Streater and Wightman 1964; Earman and Fraser 2006), tries to translate the prediction recipes which work in empirical physics into theories which are provably self-consistent mathematical theories of how to make predictions.

Perhaps the greatest breakthrough in empirical QFT since the development of canonical quantum electrodynamics (QED) in the 1950s was the new version of QED developed by David Deutsch of Oxford (Deutsch 1997), building on earlier work by Everett and Wheeler (DeWitt and Graham 1973). In a way, this was just a new interpretation of canonical QED, consistent with hard core philosophical realism (which I tend to believe but do not take as certain or a priori). But it also led to massive empirical success and useful new designs in the realm of electronics and photonics (Deutsch 1985). All of our hard core knowledge about local causal hidden variable theories in physics (Bell 1987; Clauser and Shimony 1978) is also based on experiments in the domain of QED, within the scope of Deutsch's mathematical formulation of canonical QED. In my view, a more complete understanding of the empirical realm of QED is an essential prerequisite to a reliable complete scientific understanding of how the cosmos works in broader terms.

Deutsch's version of canonical QED assumed that our cosmos is not just a curved Minkowski space-time, as in Einstein's general relativity (GR). Rather, it is an infinite dimensional "Fock space" (aka "multiverse" or "configuration space"), patched together with time in a way which makes it appear empirically that time is just another dimension even though it is not. (No, Einstein did not like this, and mathematicians still hope (Glimm and Jaffe 1981; Streater and Wightman 1964) for a more transparently relativistic version of QFT. Feynman path is transparently relativistic but not so transparently meaningful mathematically.) Using Deutsch's version of canonical QED (Deutsch 1985, 1997), it is easy enough to match the decisive Bell's theorem experiments using a measurement prediction rule which does not entail any kind of metaphysical observer.

For myself, I now give equal subjective probability to three possibilities for what the ultimate mathematical laws of physics may be:

(1) Einsteinian realism, which cannot yet be ruled out (Werbos 1973, 1989, 2008), but which needs to confront the very strong evidence for "macroscopic Schrodinger cats (Friedman and Han 2003; Abdi et al. 2016)", which implies that quantum superposition applies even to macroscopic objects like cats and humans (Nietzsche 1956) and whole planets;

(2) multiverse realism, like Deutsch's initial concept (Deutsch 1997), with or without a modified measurement theory (Werbos 2008, 2013a), with or without a four dimensional version of Fock space (Streater and Wightman 1964);

(3) something much weirder, such as cosmos as a random graph (Wolfram 2002), a "digital universe" (Bear 1993) or a kind of vast intrinsic neural network yielding strange but testable "idealist" phenomena like those in the movie Inception. I begin to see ways to do a little serious empirically grounded justice to possibilities in group (3), but there are ever so many different possibilities in group (3) that I view it as more promising to focus our efforts mainly on possibilities (1) and (2) for now. There is a huge difference between our long-term vision (subjective probabilities) of what the ultimate distant mountains might be, and our strategic sense of what we need to learn next in order to find out. In discussing this third set of possibilities, I initially referred to it as "weirdism," but admitted that the term "ultraweirdism" is more appropriate, since even Einsteinian realism predicts many things which seem weird or impossible to naïve intuition, when we consider theories which allow for macroscopic Schrodinger cats.

In truth, like De Broglie (DeBroglie and Mechanics 1960) and Sutherland (Sutherland 2017), I have put enormous effort into the search for PDE plausible as an Einsteinian "law of everything". However, no such models will be really credible in empirical physics until at least one of them fully addresses the first core empirical challenge, how to explain in 
mathematics why old QM did so well in predicting the spectrum of helium (Werbos 2013b). I have developed the new mathematics which gives at least a sketch solution to that problem (Werbos 2013c), though it requires that we explain the "pilot wave" as a kind of FM statistical wave instead of the old DeBroglie/Bohm idea of a more AM type of "linear wave." My initial analysis of the earlier work suggested at first that one of them might actually meet the most critical requirements (Werbos 2017a), but there are further requirements to fit what is known from the standard model of physics, and I have yet to publish any of the new candidate models which I hope might be sufficient (because of more urgent priorities here). In my view, we cannot create real credible physics for possibility (1) until we recruit younger and better mathematicians to this challenge; that in turn requires that we use experiments to lay to rest the legitimate concerns of the mainstream regarding the level of hope for local realistic theories of physics. Rational German existentialism also tells me that we need deeper efforts to advance possibilities (2) and (3) as well, building on what a new theory to predict quantum measurement can tell us.

Please forgive if I try to put this section into perspective by telling an old story (as old men need to do, to pass on more pure unedited first person experience). Years ago, when I organized an NSF workshop on engineering uses of neural network technology, jointly sponsored by Mexico, I asked the Mexican representative, Nydia Lara, why she wanted to use a tree as the symbol of that conference. She said: "This is the sacred tree of the Mayans, the Si'an Kaan, which grows the bridge between the peoples stuck in the mud and the people lost in the clouds." Science does need mud and it does need vision into the clouds, but a stronger bridge - the next big thing in empirical QED - is what all of physics needs most at this time, according to everything I have seen.

\section{New Experiments to Nail Down Quantum Measurement: The Next Big Need in Physics}

\section{The Core Challenge of Quantum Measurement}

Following Stapp, Von Neumann's formulation of quantum mechanics consisted of two processes used in making predictions:
Process 1: what is usually called "quantum measurement" in modern courses, comprising the eigenvector projection model of measurement, collapse of the wave function, the Born rule, etc.

Process 2: quantum dynamics, which in canonical QED is simply the "modern Schrodinger equation" (not what Schrodinger wrote!):

$\dot{\psi}=i H \psi$

Strictly speaking, QED defined in this way gives grossly inaccurate predictions in many experiments unless we add a third process, "Fermi's Golden Rule," for spontaneous emission; that process is important, but for the sake of simplicity I will say very little about it here.

Equation 1 is all we need to fully specify Process 2 in QED, if we follow David Deutsch and the original work in canonical QED (Schwinger 1958; Mandl 1959) by specifying H as the normal product form of the Maxwell-Dirac Hamiltonian. Process 1 is what has not been fully nailed down yet in experiments designed to test competing predictions of competing theories.

The confusion and uncertainty about Process 1 even in empirical physics (let alone ontological stuff) can be seen very clearly by carefully studying the basic "Bell" experiment (Fig. 1) of Clauser, Holt, Shimony, and Horn, which underlies almost everything we really know about "local, causal hidden variable theories" (Bell 1987; Clauser and Shimony 1978; Werbos 2013a).

It is well known that these experiments validate "the predictions of quantum mechanics," but how exactly were those predictions calculated in the papers which reported that result? These predictions were all based on a "measurement" of the linear polarization on both channels, but when does the wave function collapse, if it collapses at all?

Even in the practical field of Quantum Information Science and Technology (QuIST), different models are used in practice to make these kinds of predictions, and such differences have actually led to different views of what designs would actually work (Werbos 2008).

Some physicists would model the polarizer as an object which performs a unitary transformation on the incoming wave function, and assumes that a stochastic decision is made at the detector, which passes on either a " 1 " or a " 0 " based on the usual Born rule, without any human observer being

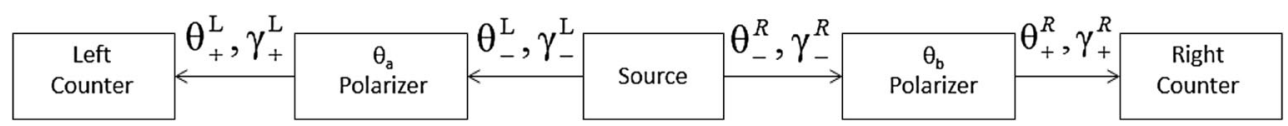

Fig. 1 Schematic of the "Bell" experiment of CHSH. The "Source" is the source of two entangled photons, a Bell state. The task is to measure R2, the rate at which photons are counted at the same time on both counters, as a fraction of R0, the rate at which pairs are produced, as a function of the angles of the polarizers (Werbos 2013a) 
needed. Others would assume that there is no collapse until a human looks at the printout. I have adapted (Werbos 2013a) the methodology which is now standard in successful, empirical quantum optics (Walls and Milburn 1994; Carmichael 1999; Mandel and Wolf 1995) by modeling the polarizer itself as a kind of stochastic object, which may input a pure wave function but outputs a density matrix $\rho$ which represents a stochastic mix of possible outgoing wave functions. The behavior of most solid objects passing on quantum states of light is modeled in detail by assuming that interactions of the light with the unknown thermodynamic state of the object itself (the reservoir) (Walls and Milburn 1994) is the source of stochasticity. This approach reproduces all the standard predictions and standard details of calculation familiar in solid empirical analysis of the Bell experiment (e.g., (Clauser and Shimony 1978; Scully and Zubairy 1997)), without any conscious observers assumed or required whatsoever.

\section{A New Approach to the Challenge of Quantum Measurement}

This leads to a new approach to the challenges of quantum measurement: the challenge of developing, testing, and refining empirical models of objects like polarizers used in quantum optics. This approach converts the metaphysical mystery of quantum measurement into an exercise in systematic device characterization, an exercise which is really crucial to our future ability to build new devices which fully exploit the potential power of quantum technology. This is not an attack on the existence of consciousness or the soul, but an effort to get past equating those things with a pair of sunglasses.

Hugh Everett (DeWitt and Graham 1973) made a great effort to simplify QED, by trying to derive Process 1 from the emergent behavior predicted by Process 2. In (Werbos 2008), I explained why such a derivation is not logically possible, because the Born rule is asymmetric in time and Eq. 1 is not. However, a fully coherent and realistic version of QED is possible, with measurement models consistent with the dynamics (potentially derivable from the dynamics), simply by modifying the measurement rules to conform with the constraints described in (Werbos 2008), which spells out my version of time-symmetric physics (not to be confused with the many other doctrines occasionally spoken about attached to those words). We have actually performed those modifications of the models for several objects, such as various types of polarizers, both at the gross level assumed by the Born rule and in continuous time models of what happens inside a polarizer.

These modified models made predictions which are distinctly different from those of the Born rule. For example, in (Werbos 2018b), I give more of the details of the predictions of canonical QED for the all-angles triphoton experiment proposed in (Deutsch 1985). Anyone who claims to believe in the
Born rule, and who acknowledges its importance, should be interested in testing those canonical predictions, even though they disagree sharply with what I predict from modified QED (MQED), QED modified by retaining Eq. 1 and the multiverse idea but modifying the models of measurement (and making it possible to derive Fermi's Golden Rule itself as a consequence of the dynamics and the boundary conditions, an aspect of canonical QED and CQED which I did not fully grasp when I wrote (Werbos 2008).)

At this writing, preliminary results have been obtained for one of the three streams of experiment and do appear to violate the Born rule; however, this kind of new direction requires great checking, scrutiny, and replication no matter how the initial results come out. For now, it is enough to say that we know these kinds of experiments are extremely important, not only for quantum optics but for future technologies using related objects like spin gates (Werbos and Dolmatova 2016), regardless of how they come out.

All of this physics does not tell us much about consciousness or the soul, topics which require much more extended discussion drawing on neuroscience, neural networks, first person psychology, and related fields (Werbos 2012, 2015, 2017b, 2018 a; Werbos and Davis 2016). The first person study of qualia in concrete useful detail can be grounded in physics, but requires many, many further concepts about emergent phenomena and about personal experience which are not an explicit part of the ultimate laws of physics.

\section{Compliance with Ethical Standards}

Conflict of Interest The authors declare that they have no conflict of interest.

Open Access This article is distributed under the terms of the Creative Commons Attribution 4.0 International License (http:// creativecommons.org/licenses/by/4.0/), which permits unrestricted use, distribution, and reproduction in any medium, provided you give appropriate credit to the original author(s) and the source, provide a link to the Creative Commons license, and indicate if changes were made.

\section{References}

Abdi, M., Degenfeld-Schonburg, P., Sameti, M., Navarrete-Benlloch, C., \& Hartmann, M. J. (2016). Dissipative optomechanical preparation of macroscopic quantum superposition states. Physical Review Letters, 116(23), 233604.

Bear, G. (1993). Moving mars, Tom Doherty Associates (NY).

Bell, J. S. (1987). The speakable and unspeakable in quantum mechanics. Cambridge U. Press.

Carmichael, H. J. (1999). Statistical methods in quantum optics 1: master equations and Fokker-Planck equations. Berlin: Springer,; Carmichael, H. J. [2007], Statistical Methods in Quantum Optics 2, Springer, New York.

Clauser, J. H., \& Shimony, A. (1978). Bell's theorem: experimental tests and implications. Reports on Progress in Physics, 41, 1881-1927. 
DeBroglie, L., Mechanics, N. W. (1960). A causal interpretation (A. Knodel \& J. C. Miller, tr). Elsevier.

Deutsch, D. (1985). Quantum theory, the Church-Turing principle and the universal quantum computer. Proceedings of the Royal Society of London A, 400(1818), 97-117 See also his receipt of the Dirac award for the invention of quantum computing: www.youtube.com/ watch? $=\mathrm{J} 7 \mathrm{HeDX}$ _ 7 Heg.

Deutsch, D. (1997). The fabric of reality: the science of parallel universes and its implications. Penguin.

DeWitt, B. S., \& Graham, N. (Eds.). (1973). The many worlds interpretation of quantum mechanics. Princeton University Press.

Dowling, J. P., Klimeck, G., \& Werbos, P. (2007). Final report on ECCS/ NSF workshop on quantum, molecular and high performance modeling and simulation for devices and systems (QMHP) arXiv: 0709.3310v2

Earman, J., \& Fraser, D. (2006). Haag's theorem and its implications for the foundations of quantum. Erkenntnis, 64(3), 305-344. https://doi. org/10.1007/s10670-005-5814-y.

Friedman, J. R., \& Han, S. (2003). Exploring the quantum/classical frontier: recent advances in macroscopic quantum phenomena. Nova Publishers.

Fromm, E. (1976). Escape from freedom (p. 1941). New York: Rinehart. Fung, Y.-L. (1967). The spirit of Chinese philosophy. Beacon Press.

Glimm, J., \& Jaffe, A. (1981). Quantum physics: a functional integral point of view. New York: Springer.

Greeley Greeley, A. M., \& McCready, W. C. (1975). Are we a nation of mystics? New York Times Magazine, 1975. Reprinted in Goleman, ed, Consciousness.

Hebb, D. O. (1949). The Organization of Behavior. Wiley.

Hegel, G. W. F. (1861). Lectures on the philosophy of history. Henry G. Bonn.

Hooft, Gerard'T (2014). "The cellular automaton interpretation of quantum mechanics. arXiv preprint arXiv: 1405.1548

Hume, R. E. (1921). In H. Milford (Ed.), The thirteen principal Upanishads: translated from the Sanskrit with an outline of the philosophy of the Upanishads and an annotated bibliography. Oxford University Press.

Kant, I. (1999). Critique of pure reason. Cambridge university press. http://www.bookwolf.com/Wolf/pdf/ImmanuelKantTheCritiqueofPureReason.pdf.

Krishna, G. (2017). Living with kundalini: the autobiography of Gopi Krishna. Shambhala Publications.

Mandel, L., \& Wolf, E. (1995). Optical coherence and quantum optics. Cambridge University Press.

Mandl, F. (1959). Introduction to quantum field theory. Wiley.

Micale, M. S., \& Micale, M. S. (2009). Hysterical men: the hidden history of male nervous illness. Harvard University Press.

Nietzsche, F. W. (1956). The birth of tragedy; and, the genealogy of morals. Anchor.

Raiffa, H. (1968). Decision analysis: introduction lectures on choices under uncertainty. Addison-Wesley.

Rand, A. (2005). Atlas shrugged. Penguin.

J. Schwinger ed. (1958). Selected papers on quantum electrodynamics, Dover.

Scully, M. O., \& Zubairy, S. (1997). Quantum Optics. Cambridge University Press.

Stapp, H. (1998). Pragmatic approach to consciousness. In K. Pribarm (Ed.), Brain and values: is a biological science of values possible. Erlbaum.

Stapp, H. P. (2004). A quantum theory of the mind-brain interface. In Mind, matter and quantum mechanics (pp. 147-174). Berlin, Heidelberg: Springer http://www.iaea.org/inis/collection/ NCLCollectionStore/_Public/22/019/22019953.pdf.
Streater, R. F., \& Wightman, A. S. (1964). PCT, Soin and Statistics and All That. Princeton University Press.

Sutherland, R. (2017). How Retrocausality Helps, arXiv: 1706.02290.

Von Neumann, J., \& Morgenstern, O. (2007). Theory of games and economic behavior (commemorative edition). Princeton: Princeton university press.

Walls, D. F., \& Milburn, G. F. (1994). Quantum Optics. New York: Springer.

Weinberg, S. (1995). The quantum theory of fields. Cambridge University Press.

Werbos, P. (1973). An approach to the realistic explanation of quantum mechanics. Nuovo Cimento Letters, 8(2), 105-109.

Werbos, P. (1989). Bell's theorem: the forgotten loophole and how to exploit it. In M. Kafatos (Ed.), Bell's theorem, quantum theory and conceptions of the universe. Kluwer.

Werbos, P. (2006). Space, ideology and the soul: a personal journey. In R. Krone (Ed.), Beyond Earth, Apogee Books. www.werbos.com/ Space_personal_Werbos.htm.

Werbos, P. J. (2008). Bell's theorem, many worlds and backwards-time physics: not just a matter of interpretation. International Journal of Theoretical Physics, 47(11), 2862-2874 (open access).

Werbos, P. (2012). Neural networks and the experience and cultivation of mind. Neural Networks, 32, 86-95.

Werbos, P. J. (2013a). Lumped Parameter Modeling of a Quantum Optics Circuit and Decisive Test for Time-Symmetric Physics, arXiv: 1309.6168

Werbos, P. J. (2013b). Solitons for describing 3-D physical reality: the current frontier. In Chaos, CNN, Memristors and beyond: A Festschrift for Leon Chua with DVD-ROM, composed by Eleonora Bilotta (pp. 448-457).

Werbos, P. ( 2013c). Extension of the Glauber-Sudarshan mapping for classical and quantum energy spectra. arXiv: 1402.5116.

Werbos, P. J. (2015). Links between consciousness and the physics of time. International IFNA -ANS Journal "Problems of nonlinear analysis in engineering systems. www.werbos.com/Mind in Time.pdf.

Werbos, P. (2017a). Preliminary evidence that a neoclassical model of physics (L3) might be correct, http://vixra.org/abs/1704.0264. But see http://vixra.org/abs/1901.0023.

Werbos, P. (2017b). Unification of objective realism and spiritual development. http://scsiscs.org/conference/index.php/ scienceandscientist/2017/paper/view/166/53.

Werbos, P. (2018a), Consciousness from AI to noosphere, plenary talk at the Science of Consciousness Conference, Tucson, Arizona, link to slides and talk posted at www.facebook.com/paul.werbos/posts/ 1924099547620453.

Werbos, P. J. (2018b). Predictions of traditional observer-free quantum mechanics for the all-angles triphoton experiment. https://doi.org/ 10.13140/RG.2.2.21773.44008R .

Werbos, P. J., and Davis, J. J. J. (2016). Regular cycles of forward and backward signal propagation in prefrontal cortex and in consciousness. Frontiers in Systems Neuroscience 10. www.ncbi.nlm.nih.gov/ pmc/articles/PMC5125075/

Werbos, P. J., \& Dolmatova, L. (2016). Analog quantum computing (AQC) and the need for time-symmetric physics. Quantum Information Processing, 15(3), 1273-1287.

Wolfram, S. (2002). A new kind of science (Wolfram Media, Inc.,). See also http://www.wolframscience.com/nksonline/toc.html.

Publisher's Note Springer Nature remains neutral with regard to jurisdictional claims in published maps and institutional affiliations. 